\title{
High-Throughput Assessment of Structural Continuity in Biologics
}

\author{
Caterina Musetti, Mark F. Bean, Geoffrey T. Quinque, Christopher Kwiatkowski, Lawrence M. Szewczuk, ${ }^{\dagger}$ \\ John Baldoni, and Matthew A. Zajac*
}

Platform Technology and Science, GlaxoSmithKline, 1250 South Collegeville Road, Collegeville, Pennsylvania 19426, United States
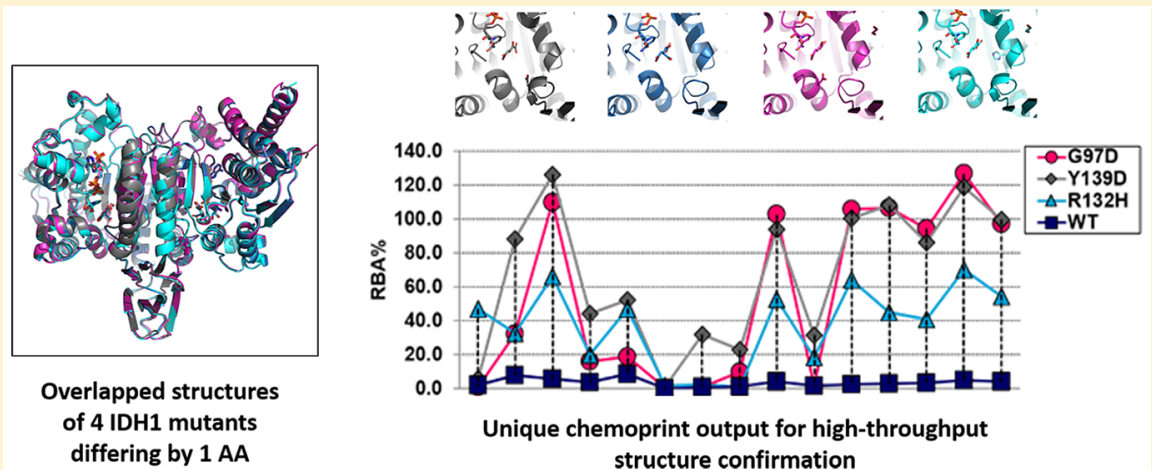

ABSTRACT: We demonstrate a high-throughput chemoprinting platform that confirms the consistency in the higher-order structure of protein biologics and is sensitive enough to detect single-point mutations. This method addresses the quality and consistency of the tertiary and quaternary structure of biologic drug products, which is arguably the most important, yet rarely examined, parameter. The method described uses specific small-molecule ligands as molecular probes to assess protein structure. Each library of probe molecules provides a "fingerprint" when taken holistically. After proof-of-concept experiments involving enzymes and antibodies, we were able to detect minor conformational perturbations between four $48 \mathrm{kDa}$ protein mutants that only differ by one amino acid residue.

$\mathrm{T}$ he recognized significance of pharmaceutical biologics lies in their ability to provide effective therapies for previously incurable conditions. This modality has developed as a major therapeutic option, and there is an ever-increasing need for constant improvement to the development paradigm, especially during manufacturing, where it is critical to assess drug quality accurately. ${ }^{1}$ This is especially important when changes are implemented to a manufacturing process, where ultrasensitive analytical methods are required to quickly assess the product and ensure the industry's high safety and efficacy standards are met. $^{2}$

Due to the structural complexity of a biologic, extensive physicochemical characterization is required involving multiple complementary and orthogonal analytical methods. ${ }^{3}$ Mass spectrometry (MS) is easily automated and has advantages of speed, specificity, and sensitivity in detecting primary amino acid sequence changes (e.g., truncation, deamination, oxidation $)^{4}$ and post-translational modifications (e.g., glycosylation, phosphorylation). ${ }^{5}$ Structure preservation, ${ }^{6}$ in particular, is of paramount importance when establishing a biologic's efficacy and safety of use. ${ }^{7}$ Due to its association to function, higherorder structural changes can result in severely diminished activity and immunogenicity. ${ }^{8}$ It is therefore not surprising that regulatory agencies have increased scrutiny around drug manufacturers' ability to confirm structural continuity between bulk samples of temporal lots. ${ }^{9}$ Unfortunately, no method currently assesses these changes in a reliable and highthroughput fashion. To address this, we have developed a high-throughput chemoprinting platform based on affinity selection mass spectrometry (ASMS) that confirms the higher-order structure of biologics (down to single-point mutations) using specific small-molecule ligands as molecular probes.

Our overall workflow (depicted in Scheme 1) relies upon ASMS, which is a high-throughput technology that directly assesses ligand/target interactions. ${ }^{10}$ The ligand is ultimately identified by its unique mass signature, and because of this, hundreds of compounds can be screened simultaneously if each ligand has a unique mass. In this process, the biologic is first incubated with a small molecule or mixture of molecules (massencoded mixture), allowing specific protein-ligand complexes to form. The molecules with weak or no affinity are removed from solution using size exclusion chromatography. This results in an eluate-containing protein and an enrichment of ligand(s) that have affinity for the biologic. Reversed-phase accurate-mass liquid chromatography-mass spectrometry (LC-MS) analysis follows, to detect the mass of the ligand(s). The amount of ligand bound (selection), normalized to the signal detected

Received: January 12, 2018

Accepted: January 25, 2018

Published: January 25, 2018 
Scheme 1. High-Level Workflow of the Chemoprinting Platform

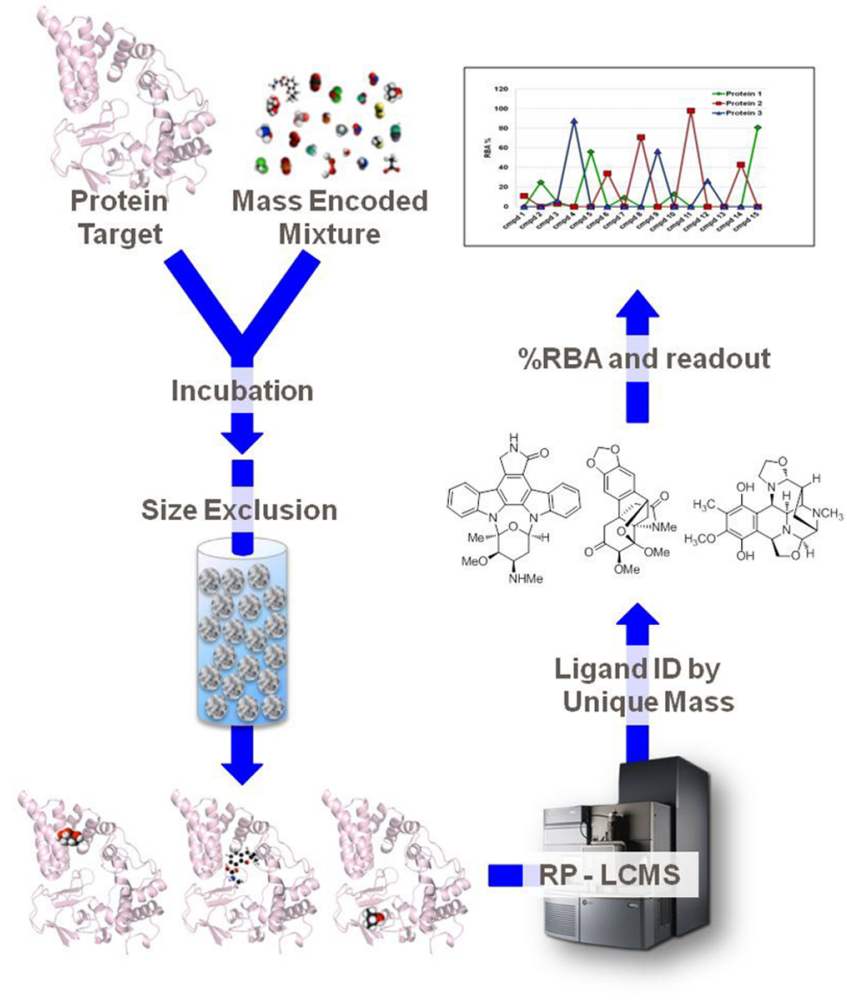

from the small molecule in the absence of the protein target (control), generates a relative binding affinity percentage (\% RBA). A higher \%RBA is, in most cases, indicative of a stronger affinity. These unique affinities (\%RBA) for a given set of ligands with a protein are specific and sensitive enough to provide a "fingerprint" when taken holistically. This method can identify perturbations, such as amino acid transpositions or higher-order structural changes. Values for \%RBA can then be graphed (Scheme 1, top right) for a given sample target with certain ligand sets. Using this readout, the protein's structural continuity can rapidly be confirmed against a fully characterized, competent protein standard.

To demonstrate the feasibility of the method we performed a number of proof-of-concept experiments using purified enzymes. Initially, we focused on identifying a library of 100 molecules that generates a specific binding profile toward four proteins belonging to distinct enzymatic classes in their native and denatured states. The library was screened as single compound per well as well as in mixtures of 10 and 25 compounds per well. Subsequently, we tested the feasibility of using a smaller set of ligands (15) to discern structural differences between three proteins sharing the same mechanism of action, but deriving from different species. The successful preliminary outcome encouraged us to form a library of ligands capable of distinguishing between three monoclonal antibodies currently in evaluation for therapeutic purposes. Finally, we further challenged the system to assess its ability to detect minor conformational perturbations between four $48 \mathrm{kDa}$ protein mutants that differ by only one amino acid residue.

\section{RESULTS AND DISCUSSION}

An initial proof-of-concept experiment was designed to test the feasibility of the method. For this experiment, four protein targets were selected which cover a range of functional and structural classes: bacterial acetyl coenzyme-A carboxylase (carboxy transferase domain, BACC), human focal adhesion kinase (FAK), human SmyD3 histone methyltransferase (SmyD3), and human Sirtuin3 (Sirt3). A customized screening library of 100 compounds was generated using available highthroughput screening data sets obtained from biochemical assays. Ligands covering a wide range of potency for each target ( $\mathrm{pIC}_{50}$ 4.5-8.9) were included, together with a few negative controls (compounds biologically inactive against all four targets). These ligand sets were sourced based on chemical diversity, the minimization of isobars, and the presence of at least one basic nitrogen atom to provide ready ionization for MS detection. As shown in the plot in Figure 1, reporting \% RBA ( $y$-axis) of every compound toward each of the four enzymes, the obtained screening library successfully generated distinctive and reproducible readouts (Table S1) that were specific for each native target. ${ }^{11}$

To evaluate the effect of higher throughput on ligand binding to a given target, the experiment for BACC was repeated by pooling the above tested library (100 compounds) in massencoded mixtures of 10 and 25 compounds per well. To avoid the likelihood of competitive binding, the mixtures were tested at a final concentration of $1 \mu \mathrm{M}$ while the protein concentration was kept at $10 \mu \mathrm{M}$. Ligand binding was preserved, and the data was reproducible (triplicate) regardless of the size of the compound pool. The \%RBA for the experiment with 25

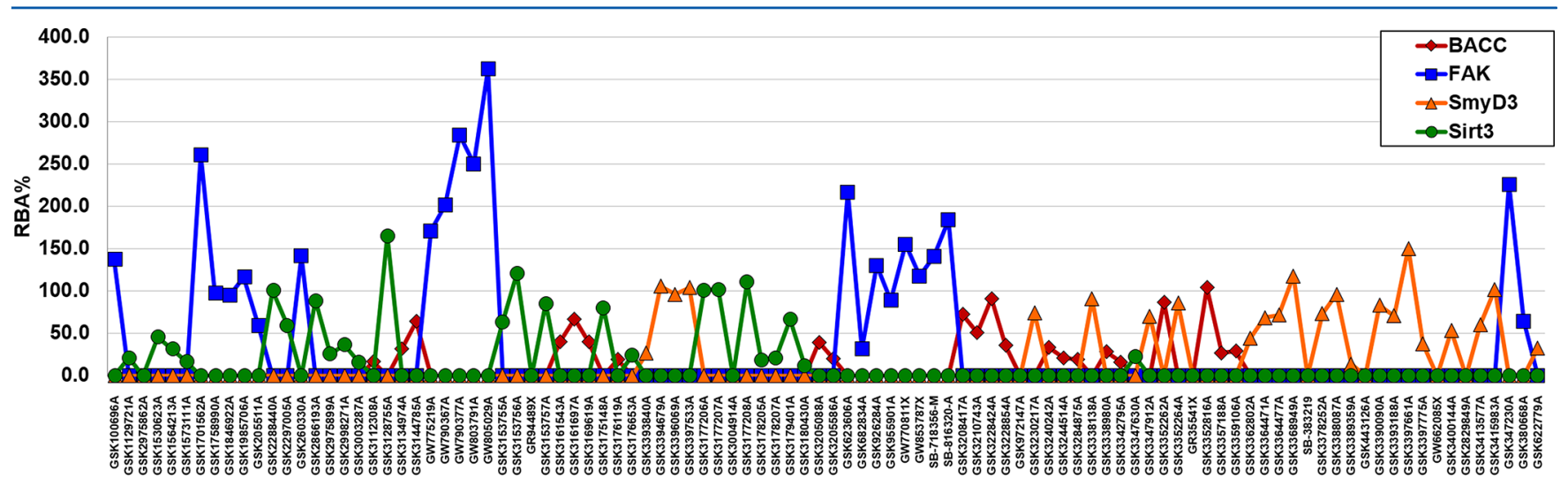

Figure 1. Graphic representation indicating the relative binding affinity percentage, \%RBA ( $y$-axis), of 100 small molecules ( $x$-axis) vs BACC (red diamonds), FAK (blue squares), SmyD3 (orange triangles), and Sirt3 (green dots). 
compounds per well is shown in Figure 2 and includes the associated error bars. The absolute \%RBA, however, varies

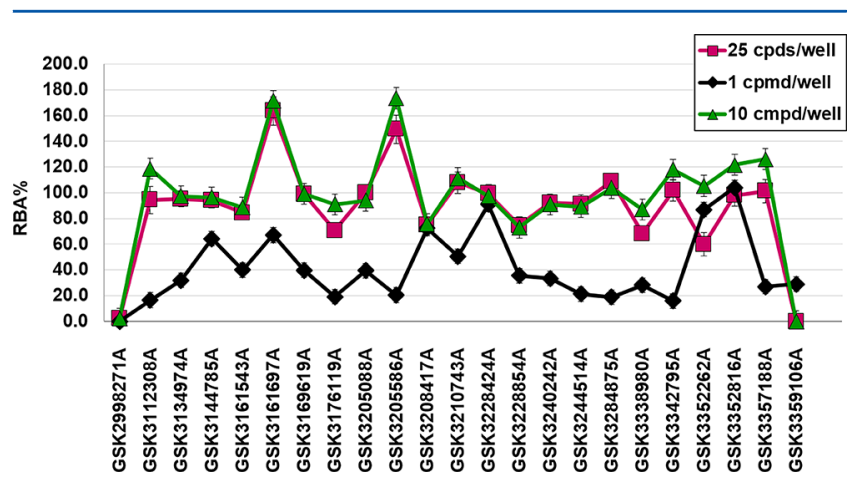

Figure 2. Graphic representation indicating the relative binding affinity percentage, \%RBA ( $y$-axis), of 23 small molecules ( $x$-axis) from a library of 100 vs BACC when the library was tested in mixtures of 25 compounds per well (four wells with 25 compounds per well).

when the protein is incubated with a single compound per well or with mixtures of 10 and 25 because the experiments differ in the ligand/protein ratio and because MS ion suppression is more likely as the number of compounds increases, thus changing the \%RBA (Figure 2). ${ }^{11}$

To test the effectiveness of our ligand set in addressing gross structural changes within the same target, we tested the 100 compound library with Sirt3 denatured with $8 \mathrm{M}$ urea. The assay produced a failed chemoprint, as expected, when compared to the authentic standard chemoprint generated with native Sirt3 (Figure 3). The same outcome was observed when an acidic solution $(\mathrm{HCl}, \mathrm{pH} 2.0)$ was used to denature Sirt3. ${ }^{11}$

Further exploring the applicability of the method, we challenged its potential to distinguish between proteins that share the same biological mechanism but originate from three different species. TrmD [t-RNA (guanine-N(1)-)-methyltransferase] was evaluated from both Hemophylus influanzae (a Gram-negative bacteria) and Streptococcus pnemoniae (a Grampositive bacteria), whereas Trm5 [t-RNA (guanine(37)-N(1)methyltransferase] was derived from Homo sapiens. Sequence alignment obtained by ClustalW indicates a $50 \%$ sequence homology between the two bacterial proteins and approximately $20 \%$ between the human and the bacterial targets. Although there is not a fundamental difference in the catalytic process between these targets, the amino acid sequence deviations may lead to alternate binding orientations of the ligands since the residues available for binding could differ. The three TrmD proteins were assessed for binding against a set of 15 ligands, which generated a unique signature in terms of binding affinity and binding intensity for each target. The graph in Figure 4 summarizes the distinct protein profile generated by

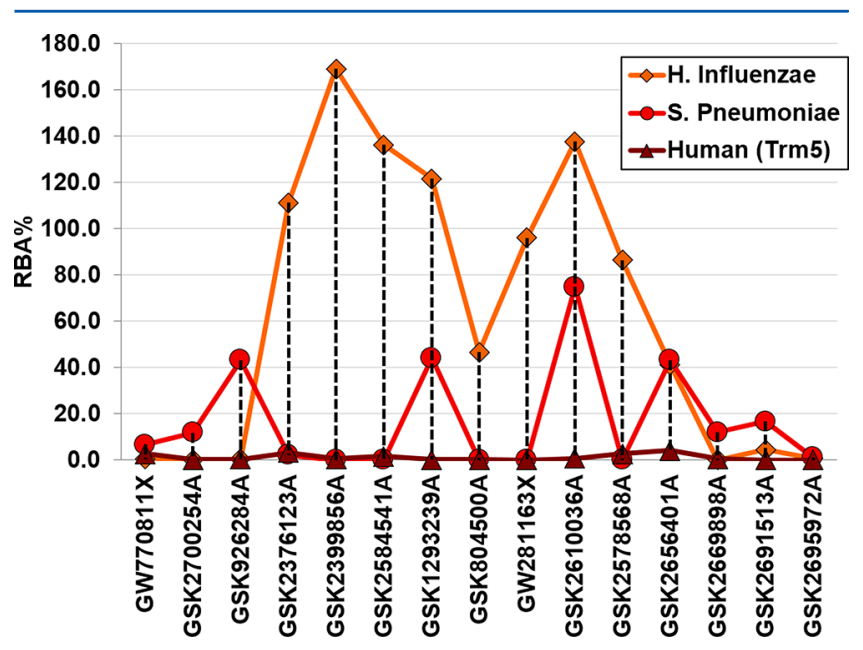

Figure 4. Graphic representation indicating the relative binding affinity percentage, \%RBA ( $y$-axis), of 15 small molecules ( $x$-axis) vs TrmD from $H$. influanzae (orange diamonds), S. pnemoniae (red dots), and human (dark red triangles).

comparing the \%RBA ( $y$-axis) of each ligand per protein species. It is noteworthy that some probes interact with the two bacterial forms with distinct affinities, while none showed affinity for Trm5.

Next, attention was turned to current antibody products in the portfolio at GlaxoSmithKline. After an exercise to identify ligands, which included screening in-house mass-encoded libraries and literature compounds, we identified a library which could easily detect differences between mepolizumab (Nucala), aHER3, and aNOGO (Figure 5). Antigens of Nucala (IL5) and aHER3 (HER3) were also included as competent members of the set. A control with a denatured antibody was included in the experiment and showed a rejected chemoprint, as expected. ${ }^{11}$ Each chemoprint is unique, and reproducibility was demonstrated with samples in triplicate.

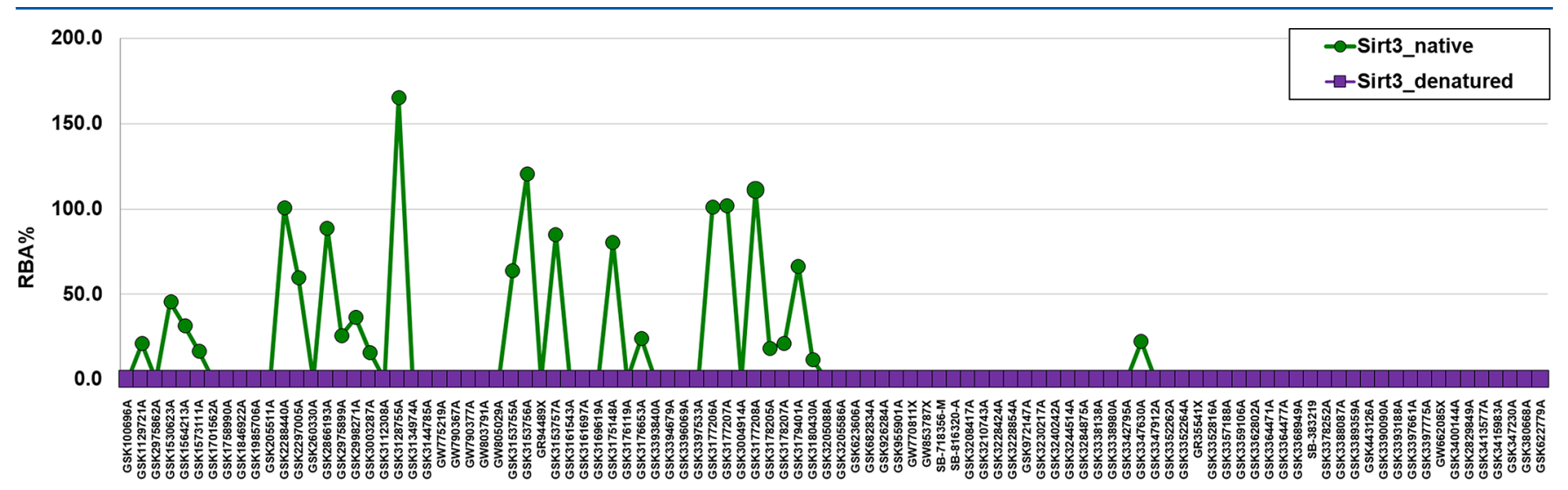

Figure 3. Graphic representation indicating the relative binding affinity percentage, \%RBA ( $y$-axis), of 100 small molecules ( $x$-axis) vs Sirtuin3 (green dots) in its native state and in its denatured state (purple squares). 


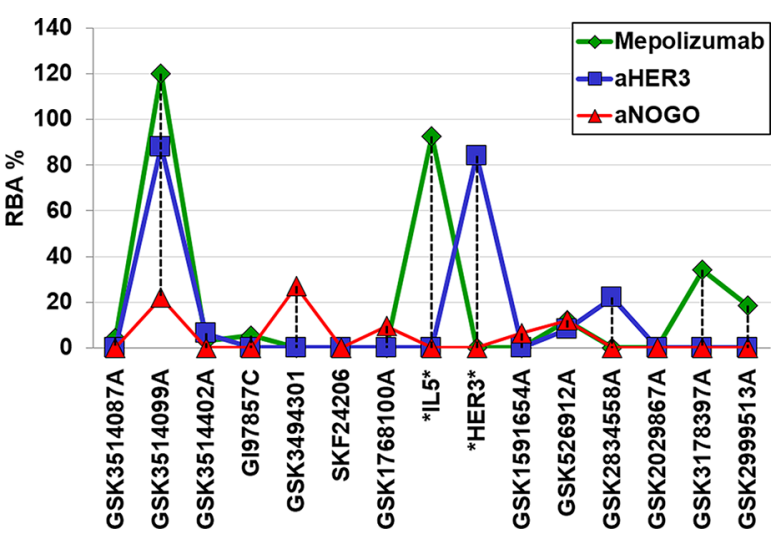

Figure 5. Graphic representation indicating the relative binding affinity percentage, \%RBA ( $y$-axis), of 15 small molecules ( $x$-axis) vs aNOGO, mepolizumab (Nucala), and aHER3.

To further challenge the robustness of our chemoprinting platform in detecting minor conformational perturbations, we focused on a single protein, isocitrate dehydrogenase 1 (IDH1) homodimer. This target was expressed as the wild type and also as three single-point mutants. Structural data obtained by superimposing the two monomers of each dimer indicate an rms difference from 0.29 to $0.40 \AA$ between $\mathrm{C} \alpha$ atoms, which is suggestive of minor structural variations between the wild type and the single mutants. ${ }^{12}$ Despite the conformational similarities between the four proteins, a library of 15 molecular probes was able to bind to each mutant with a unique overall "fingerprint". As summarized in Figure 6, while the compounds

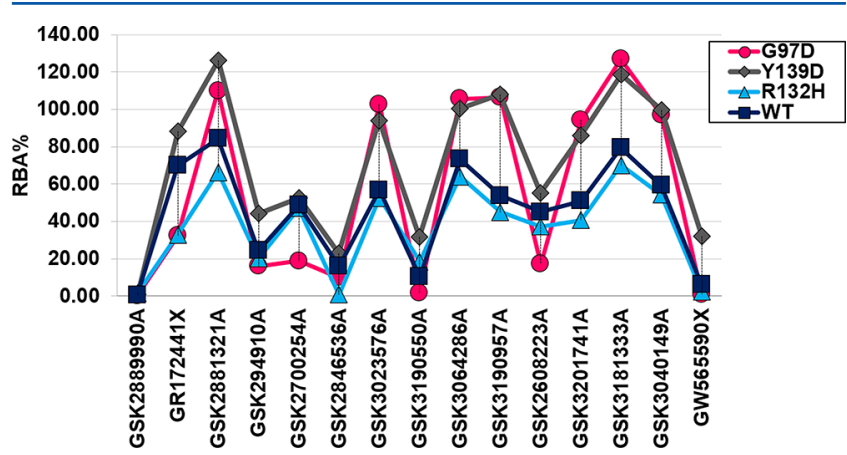

Figure 6. Graphic representation indicating the relative binding affinity percentage, \%RBA ( $y$-axis), of 15 small molecules ( $x$-axis) vs IDH1 wild type (navy blue squares) and mutants $\mathrm{R} 132 \mathrm{H}$ (cyano triangles), Y139D (gray diamonds), and G97D (magenta dots).

show very weak affinity for the wild-type protein, a distinct chemoprint is still maintained. Such a distinct profile highlights the power of this methodology in delivering a unique assay readout from proteins that differ only by a single-amino-acid transposition.

\section{CONCLUSIONS}

A biologic's lack of structural continuity between discrete samples can have dramatic physiological consequences. Because of this, regulatory agencies are demanding tighter controls on the pharmaceutical industry's ability to assess these differences to prevent potential adverse events in patients. The chemoprinting method described above allows for the confirmation of higher-order structures of a biologic, down to single-point mutations. The data provided for this high-throughput chemoprinting method establishes a foundation for its use with biologics ranging in molecular weight (MW) and biochemical function since libraries can easily be assembled to probe specific aspects of higher-order structure.

\section{EXPERIMENTAL SECTION}

Preparation of Resin for Size Exclusion Chromatography. An amount of $45 \mathrm{~g}$ of resin powder (Bio-Rad Bio-Gel P10 fine resin) was added to a solution of $10 \mathrm{mM}$ Tris, $\mathrm{pH} 7.5$ and $0.02 \%$ of sodium azide and slowly mixed until a suspension was formed. The suspension was allowed to swell overnight, and the volume was adjusted the day after to $500 \mathrm{~mL}$.

Preparation of Microtiter Plates for Affinity Selection Mass Spectrometry. A traditional ASMS experiment consists in the preparation of four types of plate $\backslash$ samples.

Size Exclusion Chromatography Plate. An amount of 130 $\mu \mathrm{L}$ of Bio-Gel P10 resin suspension was added to each well of a low protein binding Millipore Multiscreen HTS 384 well HV filter plate (referred here as "size exclusion plate") with a 0.45 $\mu \mathrm{m}$ Durapore (PVDF) membrane (no. MZHCNOW10). The plate was centrifuged at $1000 \mathrm{~g}$ for $2 \mathrm{~min}$, and the eluent flow through was discarded.

Compound Stamping. When the experiment was done using one compound per well, aliquots of $100 \mathrm{~nL}$ of $10 \mathrm{mM}$ compound in DMSO were initially transferred from a master library plate into a 384-well, clear, polypropylene assay plate using a Labcyte Echo acoustic liquid handler and backfilled with $100 \mathrm{~nL}$ of DMSO.

When the experiment was done using mixtures of 10 compounds per well, an intermediate polypropylene plate was prepared by stamping $500 \mathrm{~nL}$ of $10 \mathrm{mM}$ DMSO solution of each compound using a Labcyte Echo acoustic liquid handler to achieve a final concentration of $1 \mathrm{mM}$. Aliquots of $20 \mathrm{~nL}$ of 1 $\mathrm{mM}$ compound mixture were finally transferred from the intermediate plate into the assay plate and backfilled with 180 $\mathrm{nL}$ of DMSO.

When the experiment was done using mixtures of 25 compounds per well, an intermediate plate was prepared by stamping $200 \mathrm{~nL}$ of $10 \mathrm{mM}$ DMSO solution of each compound using a Labcyte Echo acoustic liquid handler to achieve a final concentration of $1 \mathrm{mM}$. Aliquots of $50 \mathrm{~nL}$ of $1 \mathrm{mM}$ compound mixture were finally transferred from the intermediate plate into the assay plate and backfilled with $150 \mathrm{~nL}$ of DMSO.

Assay Plate. A stock of protein solution was thawed using a water bath at room temperature, and then diluted in assay buffer to $10 \mu \mathrm{M}$. An amount of $20 \mu \mathrm{L}$ of protein solution was added to the assay plate to give a final concentration of $10 \mu \mathrm{M}$ protein and $50 \mu \mathrm{M}$ compound (when the experiment was done using one compound per well) or $1 \mu \mathrm{M}$ (when the experiment was done using mixtures). Following compound addition, the plate was centrifuged at $1000 \mathrm{~g}$ for $2 \mathrm{~min}$. The samples were allowed to incubate at room temperature for $1 \mathrm{~h}$.

No-Protein Control Plate. A "no-protein control" plate, consisting in the same set of ligands (or mixtures of ligands) at the same concentration but without protein target, was also prepared. The purpose of this control experiment was to check for compounds that are not properly retained by the size exclusion resin, resulting in potential bleed-through false positives.

At the end of the compound-protein incubation, the size exclusion plates were loaded onto a Bravo automated liquid handling platform (Agilent Technologies). The platform was used to transfer $50 \mu \mathrm{L}$ of assay buffer to the size exclusion plate, 
which was then centrifuged at $1000 \mathrm{~g}$ for $2 \mathrm{~min}$. The wash steps were repeated three additional times to allow resin equilibration. At the end of the washes, the "no-protein control" plate and the "assay" plate were loaded onto the Bravo. Amounts of $15 \mu \mathrm{L}$ of samples were transferred from the respective sample plate to the size exclusion plate. The size exclusion plates were centrifuged at $1000 \mathrm{~g}$ for $2 \mathrm{~min}$, and the flow-through fraction was collected into a 384-well clear polypropylene plate containing $5 \mu \mathrm{L}$ of acetonitrile. A concentration of $25 \%$ acetonitrile is used to induce protein precipitation. Plates were sealed and stored at $-80{ }^{\circ} \mathrm{C}$ overnight. Finally, plates were centrifuged at $2000 \mathrm{~g}$ for $30 \mathrm{~min}$ to precipitate protein prior LC-MS analysis.

Control Plate. The control plate contained the compounds by themselves to be analyzed by LC-MS. To prepare the control plate, aliquots of $20 \mathrm{~nL}$ of $10 \mathrm{mM}$ compounds in DMSO were transferred from the master library plate into a 384-well clear polypropylene plate (Greiner 781280) using a Labcyte Echo acoustic liquid handler (Labcyte, Inc.) and backfilled with $25 \mu \mathrm{L}$ of DMSO to obtain a final compound concentration of $10 \mu \mathrm{M}$.

Mixtures of compounds were prepared as outlined above. The solutions were analyzed by LC-MS.

8-Bromo-7-allyl-1,3-dimethyl xanthine was added to the completed plates to achieve a final concentration of $10 \mu \mathrm{M}$ as an internal standard, allowing us to normalize all measurements, reducing variation in injections, MS sensitivity, etc.

Liquid Chromatography-Mass Spectrometry (LCMS). All analyses were performed on a Waters Xevo G2S time-of-flight MS interfaced with a Waters Acquity I-Class UPLC and MassLynx 4.1 acquisition software. Ten microliter sample injections were made in partial loop-fill mode on a Waters SM-FL autosampler. LC separation used a Thermo Hypersil Gold $20 \mathrm{~mm} \times 2.1 \mathrm{~mm}$ column with $1.9 \mu \mathrm{m}$ particles column, heated to $55{ }^{\circ} \mathrm{C}$. The mobile phase consisted of water (solvent A) and acetonitrile (solvent B), each containing $0.1 \%$ formic acid. The LC method consisted of a linear gradient at $1.4 \mathrm{~mL} / \mathrm{min}$ from $1 \%$ to $99 \% \mathrm{~B}$ over $1.8 \mathrm{~min}$, a hold for 0.1 min, and back to $1 \% \mathrm{~B}$ in $0.01 \mathrm{~min}$. Autosampler time between injections substituted for column re-equilibration. Approximately one-third of the $1.4 \mathrm{~mL} / \mathrm{min}$ flow was directed into the mass spectrometer, and the first $0.1 \mathrm{~min}$ of the gradient containing salts was diverted away. The MS source was operated in positive-ion, centroided acquisition mode with a source temperature of $150{ }^{\circ} \mathrm{C}$, capillary voltage of $1 \mathrm{kV}$, cone voltage of $80 \mathrm{~V}$, desolvation temperature of $500{ }^{\circ} \mathrm{C}$, cone gas of $175 \mathrm{~L} / \mathrm{h}$, and desolvation gas of $1000 \mathrm{~L} / \mathrm{h}$. A leucine/ enkephalin lock-mass solution was infused to apply automated mass correction to all spectra acquired from 115 to $1300 \mathrm{~m} / z$ in $0.1 \mathrm{~s}$.

Data processing was accomplished with custom, in-house software according to the following automated algorithm. The mass spectrometric, smoothed, extracted ion chromatogram (XIC) peak areas and heights for each compound were normalized across plates for an experiment using the peak areas and heights for the internal standard. The relative binding affinity percentage (\%) was calculated as the percentage normalized XIC peak area of a compound in the assay plate divided by the normalized XIC peak area of the same compound in the control plate.

\section{ASSOCIATED CONTENT}

\section{Supporting Information}

The Supporting Information is available free of charge on the ACS Publications website at DOI: 10.1021/acs.analchem.8b00180.

Relative binding affinity percentages and structures of the compound sets used to generate the chemoprints (PDF)

\section{AUTHOR INFORMATION}

\section{Corresponding Author}

*E-mail: Matthew.A.Zajac@gsk.com.

\section{Present Address}

${ }^{\dagger}$ L.M.S.: Janssen Pharmaceuticals, Molecular and Cellular Pharmacology, 1400 McKean Rd., Spring House, PA 19477.

\section{Notes}

The authors declare no competing financial interest.

\section{ACKNOWLEDGMENTS}

We thank Joseph Kozole for contributing to development of the ASMS method, Amy Taylor, Patrick McDevitt, Hongwei Qi, and Patti McCormick for providing protein samples, and Jeff Gross, Stan Martens, Roland Annan, and Nestor Concha for helpful discussions.

\section{REFERENCES}

(1) Ramanan, S.; Grampp, G. BioDrugs 2014, 28, 363.

(2) Grampp, G.; Ramanan, S. BioDrugs 2013, 27, 305.

(3) (a) Wang, X.; An, Z.; Luo, W.; Xia, N.; Zhao, Q. Protein Cell 2018, 9, 74. (b) Fekete, S.; Gassner, A. L.; Rudaz, S.; Schappler, J.; Guillarme, D. TrAC, Trends Anal. Chem. 2013, 42, 74. (c) Ko, S. H.; Chandra, D.; Ouyang, W.; Kwon, T.; Karande, P.; Han, J. Nat. Nanotechnol. 2017, 12, 804.

(4) (a) Gahoual, R.; Busnel, J. M.; Beck, A.; Francois, Y. N.; LeizeWagner, E. Anal. Chem. 2014, 86, 9074. (b) Rosati, S.; Yang, Y.; Barendregt, A.; Heck, A. Nat. Protoc. 2014, 9, 967.

(5) (a) Kannicht, C.; Fuchs, B. Molecular Biomethods Handbook; Humana Press: Totowa, NJ, 2008. (b) Witze, E. S.; Old, W. M.; Resing, K. A.; Ahn, N. G. Nat. Methods 2007, 4, 798. (c) Carr, S. A.; Annan, R. S.; Huddleston, M. J. In Mass Spectrometry: Modified Proteins and Glycoconjugates; Burlingame, A. L., Ed.; Academic Press: New York, 2005; pp 82-115.

(6) (a) Engen, J. R.; Smith, D. L. Methods Mol. Biol. 2000, 146, 95. (b) Wei, H.; Mo, J.; Tao, L.; Russell, R. J.; Tymiak, A. A.; Chen, G.; Iacob, R. E.; Engen, J. R. Drug Discovery Today 2014, 19, 95. (c) Tsuchida, D.; Yamazaki, K.; Akashi, S. Pharm. Res. 2016, 33, 994. (d) Krishna, M. M. G.; Hoang, L.; Lin, Y.; Englander, S. W. Methods 2004, 34, 51. (e) Takamoto, K.; Chance, M. R. Annu. Rev. Biophys. Biomol. Struct. 2006, 35, 251. (f) Wang, L.; Chance, M. R. Anal. Chem. 2011, 83, 7234 .

(7) (a) Ratanji, K. D.; Derrick, J. P.; Dearman, R. J.; Kimber, I. J. Immunotoxicol. 2014, 11, 99. (b) Jefferis, R. J. Immunol. Res. 2016, 2016, 5358272

(8) (a) Wadhwa, M.; Thorpe, R. Drugs 2009, 12, 440. (b) Stas, P.; Lasters, I. Drugs 2009, 12, 169.

(9) Allison, G.; Cain, Y. T.; Cooney, C.; Garcia, T.; Bizjak, T. G.; Holte, O.; Jagota, N.; Komas, B.; Ko-rakianiti, E.; Kourti, D.; Madurawe, R.; Morefield, E.; Montgomery, F.; Nasr, M.; Randolph, W.; Robert, J. L.; Rudd, D.; Zezza, D. J. Pharm. Sci. 2015, 104, 803.

(10) (a) Zehender, H.; Mayr, L. M. Expert Opin. Drug Discovery 2007, 2, 285. (b) O'Connell, R. N.; Ramsay, J.; Rieth, S. F.; Shapiro, M. J.; Stroh, J. G. Anal. Chem. 2014, 86, 7413. (c) Annis, D. A.; Nazef, N.; Chuang, C.; Scott, M. P.; Nash, H. M. J. Am. Chem. Soc. 2004, 126, 15495. (d) Deng, Y.; Shipps, G. W.; Cooper, A.; English, J. M.; Annis, D. A.; Carr, D.; Nan, Y.; Wang, T.; Zhu, H. Y.; Chuang, C.; 
Dayananth, P.; Hruza, A. W.; Xiao, L.; Jin, W.; Kirschmeier, P.;

Windsor, W. T.; Samatar, A. J. Med. Chem. 2014, 57, 8817.

(11) See the Supporting Information for the complete data sets.

(12) Rendina, A. R.; Pietrak, B.; Smallwood, A.; Zhao, H.; Qi, H.; Quinn, C.; Adams, N. D.; Concha, N.; Du-raiswami, D.; Thrall, S. H.; Sweitzer, S.; Schwartz, B. Biochemistry 2013, 52, 4563. 